\title{
LA REVISTA PERUANA DE MEDICINA EXPERIMENTAL Y SALUD PÚBLICA AL TÉRMINO DEL 2018 Y SU RESPONSABILIDAD PARA EL PRÓXIMO AÑO
}

\author{
THE PERUVIAN EXPERIMENTAL MEDICINE AND PUBLIC HEALTH JOURNAL ATTHE END \\ OF 2018 AND ITS RESPONSIBILITY FOR THE COMING YEAR
}

Lely Solari 1,a, Zuño Burstein 1,b

Este año se ha celebrado el cuarenta aniversario de la Conferencia Internacional sobre Atención Primaria de Salud de Alma Ata (Kazajstán, setiembre de 1978) y hemos sufrido, como una triste e irónica coincidencia, la muerte el día 4 de noviembre del Dr. David Tejada de Rivero, el único peruano que ha ocupado el cargo de Sub-Director General de la Organización Mundial de la Salud (OMS), y quien fuera coordinador de dicha conferencia. En este número publicamos su último aporte a la producción científica peruana, a manera de homenaje póstumo ${ }^{(1)}$.

Al final del 2018, la Revista Peruana de Medicina Experimental y Salud Pública (RPMESP) llega con un tema vigente y de actualidad «Una Salud». La RPMESP ha cumplido todos sus objetivos y metas, gracias al invalorable y destacado esfuerzo del grupo humano que constituye un núcleo constante de trabajo conformado por el director, editor general, editor científico, editores adjuntos, asistente editorial, y algunos miembros del comité editor.

Cabe destacar que, en su calidad de órgano oficial de difusión científica del Instituto Nacional de Salud (INS), brazo científico del Ministerio de Salud del Perú, la RPMESP ha venido fomentando la discusión académica en temas relevantes en salud pública que son presentados, en las ya tradicionales, Conferencias Conjuntas con la Academia Nacional de Medicina. Estas reuniones cuentan con la participación de autoridades y expertos en cada uno de los temas y generan conclusiones y recomendaciones que se hacen llegar a los responsables temáticos gubernamentales.

Debemos reconocer que la RPMESP no es ajena a la profunda crisis de identificación institucional que sufre nuestro país, en el sentido que no todos los editores invitados, ni todos los miembros del Comité Editor o del Consejo Consultivo, cumplieron óptimamente los encargos asignados. Tenemos la esperanza de que esta situación se revierta en el próximo año. Como alternativa de solución hemos acordado cambiar de estrategia en la captación de artículos para los temas escogidos, y que sean miembros del núcleo de la RPMESP quienes asuman la labor de fomentar la recolección de los mismos, y coordinar con los autores de la sección simposio el envío de sus manuscritos.

En relación a la crisis a nivel país, la cual compromete a todos los poderes del Estado y que hemos reseñado en editoriales previas ${ }^{(2-4)}$, aún se encuentra en vías de resolución gracias a los esfuerzos de destacados profesionales en algunas instancias. Tanto la educación como la salud pública del Perú no han sido ajenas a la crisis existente, en la actualidad observamos una preocupante caída en la calidad de la atención médica ${ }^{(5)}$, agravada por la mercantilización de la medicina, la pobre adherencia de nuestros profesionales a las prácticas clínicas recomendadas, la enorme congestión en los servicios de salud (6), la deficiente formación de recursos humanos y la corrupción. El Ministerio de Salud, incluyendo a SUSALUD y la Superintendencia Nacional de Educación (SUNEDU) son las llamadas a hacer conocer esta situación a la sociedad y autoridades para poder corregirla.

Volviendo al tema del Dr. Tejada y la atención primaria de salud que él siempre defendió, vemos que persisten brechas similares a las que existían en el siglo XX, y están pendientes los arreglos dirigidos a fortalecer el nivel primario

\footnotetext{
Instituto Nacional de Salud. Lima, Perú.

Miembro del Comité editorial de la Revista Peruana de Medicina Experimental y Salud Pública; ${ }^{\text {b }}$ director de la Revista Peruana de Medicina Experimental y Salud Pública.

Recibido: 12/12/2018 Aprobado:12/12/2018 En línea: 21/12/2018
}

Citar como: Solari L, Burstein Z. La Revista Peruana de Medicina Experimental y Salud Pública al término del 2018 y su responsabilidad para el próximo año. Rev Peru Med Exp Salud Publica. 2018;35(4): 561-2. doi:10.17843/rpmesp.2018.354.4113 
sin descuidar la calidad de atención en los otros niveles, como mejorar el intercambio prestacional entre nuestros subsistemas, homogenizar la atención clínica mediante guías de práctica y resolver el desabastecimiento crónico de nuestros servicios de salud. Es decir, hay problemas permanentes e irresueltos en las últimas décadas cuyo abordaje es cada vez más urgente.

Por otro lado, los retos asociados a la globalización, cambio climático y desastres naturales, migraciones poblacionales y patógenos emergentes también ejercen una presión adicional sobre el sistema de salud y debemos darles la atención e importancia que merecen, sobre todo desde el punto de vista preventivo. Por ejemplo, el próximo año se espera la visita nuevamente del Fenómeno del Niño, con sus ya conocidas consecuencias sobre la salud de la población.

Tomando en cuenta todas estas consideraciones, el comité editorial ha visto por conveniente abordar en la temática de los números del 2019, desde retos que han quedado perennes en nuestro sistema de salud, hasta los nuevos desafíos que deberemos enfrentar. En el primer número se tocará el tema de la «Formación de profesionales de la salud», tema que abrirá la discusión sobre acreditación de universidades, formación en ética, nuevas estrategias pedagógicas y de calificación, entre otros puntos. En el segundo número se abordará el tema de la «Atención médica en el Perú», que esperemos incluya contribuciones que toquen los aspectos de financiamiento, gobernanza, calidad, incluso las redes integradas. El tercer número tratará sobre un nuevo desafío: la «Migración poblacional y salud pública», muy vigente en los últimos años. Finalmente, el último número tratará sobre «Higiene y salud oral», un tema que ha sido postergado en nuestro país.

Aprovechamos la oportunidad para hacer un llamado a la comunidad científica y académica para brindar sus aportes a estos números. Tanto los institutos de investigación como las universidades, los establecimientos de salud públicos y privados y los investigadores independientes, nacionales e internacionales, están invitados a enviar sus contribuciones en estos temas a nuestra revista. Priorizaremos los artículos originales, de simposio y de revisión en estas áreas.

La RPMESP seguirá cumpliendo su rol estratégico de ser la revista médico-científica peruana indizada a más bases de datos internacionales. Para el 2019 prevemos ser, cada vez más, una tribuna abierta para abordar temas de interés en la agenda pública y en alianza con otras instituciones como la Academia Nacional de Medicina, promover el intercambio y discusión en beneficio de la salud del pueblo peruano con énfasis en la atención centrada en la persona, y con el más alto respeto a los estándares científicos y éticos.

\section{REFERENCIAS BIBLIOGRÁFICAS}

1. Cuarenta años despues de Alma Ata: la actualidad de sus conceptos fundamentales. Rev Peru Med Exp Salud Publica. 2018;35(4): 1-3. doi:10.17843/rpmesp.2018.354.3902.

2. Burstein Z, Romaní F. La Revista Peruana de Medicina Experimental y Salud Pública en el contexto político nacional. Rev Peru Med Exp Salud Publica. 2018;35(1):5-6.

3. Burstein Z. Apreciación crítica a la gestión 2016 y perspectivas al nuevo año 2017 de la Revista Peruana de Medicina
Experimental y Salud Pública. Rev Peru MedExp Salud Publica. 2017;34(1):7-10. doi: 10.17843/rpmesp.2017.341.2761.

4. Burstein Z, Cabezas C. La Revista Peruana de Medicina Experimental y Salud Pública continúa al servicio de la salud pública del Perú y Latinoamérica. Rev Peru Med Exp Salud Publica. 2016;33(1):10-2.

5. Valenzuela-Oré F, Romaní-Romaní F, Monteza-Facho BM, Fuentes-Delgado D, Vilchez-Buitron E, Salaverry-García O. Prácticas culturales vinculadas al cuidado de la salud y percepción sobre la atención en establecimientos de salud en residentes de centros poblados alto-andinos de Huancavelica, Perú. Rev Peru Med Exp Salud Publica. 2018;35(1):84-92

6. Timaná R, Alva-Díaz C, Suárez V, Pi mentel P, Dongo V. Características y calidad de las guías de práctica clínica en el Seguro Social de Salud del Peru. Semergen. 2018;44(8):549-556. pii: S11383593(18)30317-4. doi.org/10.1016/j. semerg.2018.06.002. 